\title{
Ultrastrong regulation effect of electric field on cyclo[18]carbon: Phenomenon and essence
}

\author{
Tian $\mathrm{Lu},{ }^{*}$ Qinxue Chen \\ Beijing Kein Research Center for Natural Sciences, Beijing 100022, P. R. China \\ (http://www.keinsci.com) \\ * Correspondence author. E-mail: sobereva@sina.com
}

ORCiD

Tian Lu: 0000-0002-1822-1229

Qinxue Chen: 0000-0003-0155-2387

\begin{abstract}
Cyclo[18]carbon has a very unique geometry and electronic structure. Based on quantum chemistry calculations, we found that external electric field (EEF) has an ultrastrong regulation effect on various aspects of the cyclo[18]carbon. The main findings are as follows: (1) The EEF evidently affects geometric structure of the cyclo[18]carbon, and the ring is greatly elongated when EEF exceeds 0.02 a.u. (2) The EEF causes a huge polarization in the charge distribution of the system, and large EEF can even make some in-plane $\pi$ electrons nearly detached from the system (3) Presence of EEF significantly reduces HOMO-LUMO gap (4) The EEF not only greatly changes the position and intensity of the original absorption peak of the cyclo[18]carbon in the ultraviolet region, but large EEF also leads to a new absorption band in the visible light range and thus makes the cyclo[18]carbon show color. We made an in-depth explanation and discussion of the above phenomena from the aspects of energy, electronic structure, and atomic force. Furthermore, we demonstrated that it is feasible to equivalently apply strong EEF on the cyclo[18]carbon via a purely chemical and thus a more easily achieve way, namely introducing divalent alkaline earth metal cation. This work is not only of scientific interest in the interplay between the EEF and the cyclo[18]carbon, but may also open a novel path to explore different applications of this unusual molecule.
\end{abstract}

Keywords: cyclo[18]carbon; electric field; quantum chemistry; atomic force; bond length alternation; HOMO-LUMO gap; absorption spectrum; excited state; dipole moment; alkaline earth metal 


\section{Introduction}

Cyclo[18]carbon is a circular molecule purely composed of 18 sp-hybridized carbon atoms, it possesses 18 in-plane and 18 out-of-plane globally delocalized $\pi$ electrons. Due to the quite unusual geometric and electronic structures of the cyclo[18]carbon, this new member of carbon allotrope family has received great attentions since its first experimental observation in condensed phase in 2019. ${ }^{[1-3]}$ Significant theoretical efforts have been devoted to explore its characteristics and potential applications, ${ }^{[4-11]}$ and recently we have comprehensively investigated its properties from different aspects, including bonding nature, aromaticity, electronic and vibrational spectra, non-linear optical property, intermolecular interaction, dynamic behavior and so on. ${ }^{[12-16]}$

It is well known that external electric field (EEF) often has a great influence on chemical systems, and this has always been a hot research field. ${ }^{[17-25]}$ Interested readers are suggested to refer to the reviews by Shaik et al. in recent years and the references cited therein. ${ }^{[17,25,26]}$ The influence of EEF on pure carbon system, including fullerene, graphite, nanotube and their derivatives has been studied in many articles. ${ }^{[27-32]}$ Due to the high flexibility of the cyclo[18]carbon as well as its global conjugation character, it is expected that EEF should strongly affect its geometry, electronic structure and spectra. The aim of this work is to unveil the electric field regulation effect on the cyclo[18]carbon based on density functional theory (DFT) calculation and wavefunction analysis, we will focus on analyzing geometry deformation, electron polarization, atomic force, variation of HOMO-LUMO gap and optical absorption due to the presence of EEF. Not only observed phenomena will be described, but also underlying natures will be discussed. In addition, we will explore the possibility of equivalently applying EEF in a chemical way, which may be technically easier to achieve, namely adding monovalent and divalent cations to the cyclo[18]carbon. This research is not only of theoretical interest, but also opens a new promising way to explore the practical application of this novel system.

\section{Computational Details}

In this work, all quantum chemistry calculations were conducted by Gaussian 16 A.03 program $^{[33]}$ with $\omega \mathrm{B} 97 \mathrm{XD}$ exchange-correlation functional ${ }^{[34]}$ in combination with $6-311+\mathrm{G}(2 \mathrm{~d})$ basis set. ${ }^{[35,36]}$ We have demonstrated that $\omega \mathrm{B} 97 \mathrm{XD}$ is highly reliable for representing the cyclo[18]carbon. Diffuse function was added because it is important for faithfully representing the heavily polarized electrons under EEF. We have confirmed that all structures involved in this study correspond to minima on potential energy surface because no imaginary frequency exists. The Cartesian coordinates of the structures optimized under different magnitudes of EEF have been provided in the supplemental material. All analysis works, including evaluating atomic charges, calculating electron localization function, decomposition analysis of dipole moment, orbital 
localization, bond length and angle variation analyses, generating density difference and local integral curve, hole-electron analysis, as well as simulation and decomposition of absorption spectra, were all realized via Multiwfn 3.7 code $^{[37]}$ developed by us. Molecular structure maps were plotted by GaussView 6.0.16 ${ }^{[38]}$ software. All isosurface maps were drawn by VMD 1.9.3 ${ }^{[39]}$ based on the grid data generated by Multiwfn. Atomic force maps were plotted by VMD based on the force vectors extracted from output file of "force" task of Gaussian.

The EEF was applied via "field" keyword in Gaussian program. We have noticed that the sign convention of EEF employed in Gaussian is opposite to the general physical definition. ${ }^{[25]}$ In the whole article we follow common physical convention of the EEF direction. Note that EEF of 1 a.u. corresponds to $51.4 \mathrm{~V} / \AA$, and $1 \mathrm{~V} / \AA$ is approximately equal to 0.02 a.u. In this study, the maximal EEF we considered is 0.029 a.u., because self-consistent field iteration cannot get converged if further enhancing the field, and as will be shown later, this field strength actually corresponds to the limit of causing electron detachment.

\section{Results and Discussion}

\section{Structure deformation induced by external electric field}

We optimized geometry of the cyclo[18]carbon under EEFs of different strengths. Fig. 1 clearly illustrates how EEF deforms this molecule, and the map showing full variation of the length of longest axis $(D)$ of the ring with respect to EEF strength is given as Fig. S1. From the figures and $D$ values it can be seen that applying EEF can effectively elongate the carbon ring along the direction of EEF, and the stronger the field, the larger the deformation. If the field strength is increased to 0.020 a.u. (about $1 \mathrm{~V} / \AA$ ) or higher, the ring will become obviously oval. Note that no matter how strong the EEF is, the ring always remains exactly planar. Fig. 1 also reveals that EEF lowers symmetry of the cyclo[18]carbon from its ideal $D_{9 h}$ point group. ${ }^{[14]}$ At $E E F=0.029$ a.u., the cyclo[18]carbon has deformed to a structure that nearly satisfies $\mathrm{C}_{2 \mathrm{v}}$ symmetry. 


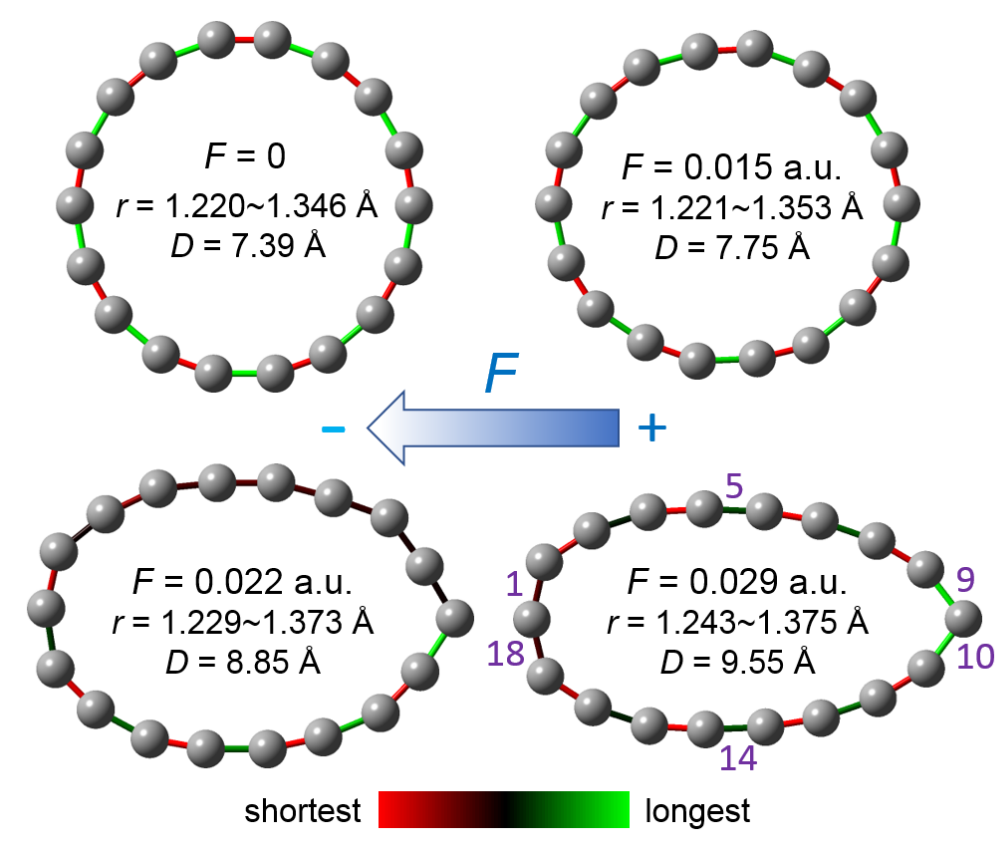

Fig. 1 Optimized geometry of cyclo[18]carbon under four representative strengths of EEF. $r$ stands for bond length. $D$ denotes the length of the longest axis. Bonds are colored according to the color bar to reflect length difference. Bond numbering is shown as purple texts.

To quantitatively study influence of EEF on bond lengths and bond angles of the cyclo[18]carbon, we plotted bond length variation and bond angle variation maps, see Fig. 2 and Fig. S2, respectively. From Fig. 2 it can be seen that when EEF is not strong, for example 0.015 a.u., the bond lengths are not notably changed compared to when there is no EEF, and the bond length fluctuates regularly along the ring. If the EEF is increased to an evidently larger value, for example, 0.0225 a.u., the bond length alternation (BLA) character will be significantly suppressed over a large part of the rings, namely from bonds 3 to 9. At the limit EEF strength (0.029 a.u.), global BLA character emerges again while the extent is generally lower than the case without EEF. It is particularly worth to note that bonds 9 and 10 become significantly longer than any other bond at $\mathrm{EEF}=0.0290$ a.u., implying that they have been greatly weakened. To further confirm this point, we calculated Laplacian bond orders $(\mathrm{LBO})^{[40]}$ for the cyclo[18]carbon and presented the result in Fig. S3. It was proven that LBO has very good correlation with bond dissociation energy for C-C type of bonds. Fig. S3 demonstrates that bonds 9 and 10 are indeed the weakest ones among all C$\mathrm{C}$ bonds at EEF of 0.029 a.u. 


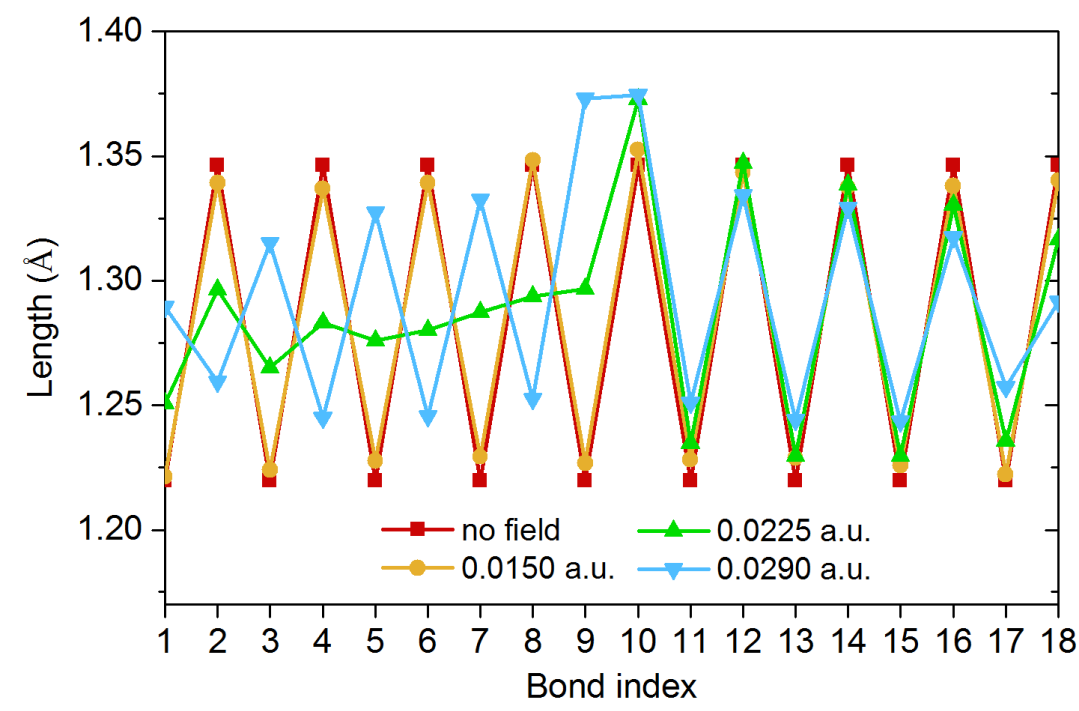

Fig. 2 Variation of bond length of cyclo[18]carbon under different magnitudes of EEF. See Fig. 1 for bond numbering.

Considering that applying EEF in different directions that parallel to the ring may result in different structures, we also tentatively applied parallel EEF in the direction perpendicular to a C-C bond, see Section S1 for details. However, the final geometry without imaginary frequency has slightly higher energy than the one when EEF is applied in the way illustrated in Fig. 1. Therefore in this work we do not consider applying EEF in other directions.

\section{Electric field effect on electronic structure}

Next, we study how EEF influences electronic structure of the cyclo[18]carbon. Dipole moment is the most straightforward index of quantifying extent of polarization due to EEF, therefore we calculated the component of dipole moment of the cyclo[18]carbon along the direction of applied EEF, see Fig. 3. It is already known that this unique system contains three sets of electrons, ${ }^{[14]}$ namely 18 out-of-plane $\pi$ electrons $\left(\pi_{\text {out }}\right), 18$ in-plane $\pi$ electrons $\left(\pi_{\text {in }}\right)$, and 72 other electrons (36 $1 s$ core electrons and $36 \sigma$ bond electrons). In order to have a deep understanding of the response of the cyclo[18]carbon to EEF, it is crucial to examine respective contributions from the three kinds of electrons. The dipole moment of the cyclo[18]carbon may be expressed as follows

$$
\boldsymbol{\mu}=\sum_{A} Z_{A} \mathbf{R}_{A}-\sum_{i \in \pi_{\text {out }}} \eta_{i}\left\langle\varphi_{i}|\mathbf{r}| \varphi_{i}\right\rangle-\sum_{j \in \pi_{\text {in }}} \eta_{j}\left\langle\varphi_{j}|\mathbf{r}| \varphi_{j}\right\rangle-\sum_{k \in \text { other }} \eta_{k}\left\langle\varphi_{k}|\mathbf{r}| \varphi_{k}\right\rangle
$$

where $A$ and $i$ loop over all atoms and occupied orbitals, respectively. $Z$ and $\mathbf{R}$ denote nuclear charge and position, respectively. $\mathbf{r}$ is electron position and $\eta$ is orbital occupation number. After properly translating the system to eliminate the contribution from the nuclear charge part, we computed contributions from $\pi_{\text {out }}, \pi_{\text {in }}$ and other electrons and plotted them as Fig. 3. 


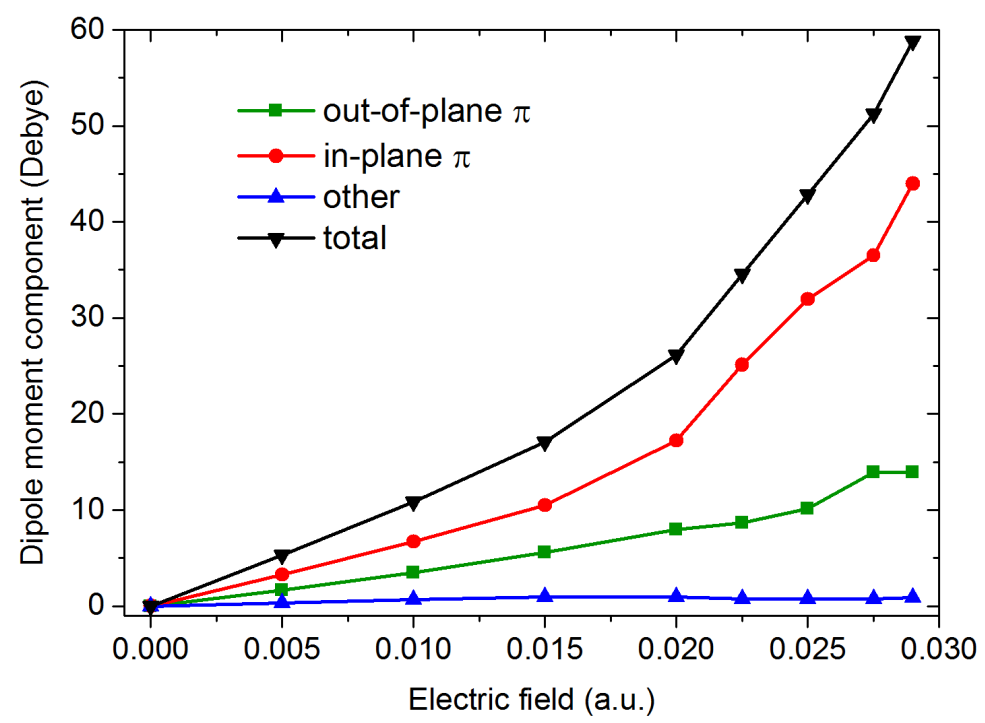

Fig. 3 Magnitude of dipole moment of cyclo[18]carbon along direction of EEF. Contributions from outof-plane $\pi$ electrons, in-plane $\pi$ electrons and other electrons are given separately, "total" corresponds to total dipole moment and equals to the sum of the three contributions.

The cyclo[18]carbon itself has zero permanent dipole moment due to its centrosymmetric geometry. From Fig. 3 it can be seen that the dipole moment of this system induced by EEF increases rapidly with increase of EEF strength, and the slope becomes much larger after EEF $=0.020$ a.u. It can be found that $\pi_{\text {out }}, \pi_{\text {in }}$ and other electrons show strikingly different response behaviors to EEF. The electrons forming 18-center in-plane $\pi$ bonds are most sensitive to EEF, and it is the major contributor to the total induced dipole moment. In contrast, although the $\pi_{\text {out }}$ electrons also globally delocalize over the ring like the $\pi_{\text {in }}$ electrons, they are significantly less polarizable by EEF. The polarizability of other electrons can be fully ignored, as the blue line in Fig. 3 clearly indicates that their contribution to dipole moment is negligible even under a fairly large EEF. This observation is easily understandable since they occupy highly localized orbitals, namely core and $\sigma$ orbitals.

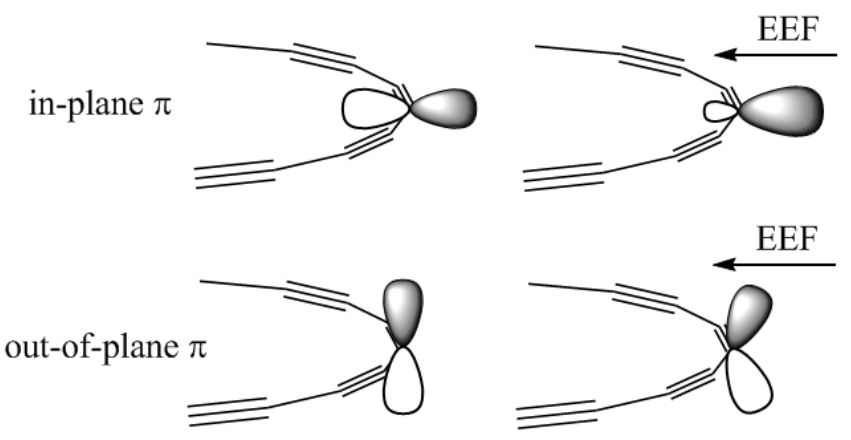

Fig. 4 Illustration of polarization of a $p$ type atomic orbital of a carbon atom under EEF.

Why $\pi_{\text {in }}$ electrons are more polarizable than $\pi_{\text {out }}$ electrons by the EEF applied parallelly to the 
ring? Although the reason is seemingly complicated, we try to explain it to some extent from the shape of atomic orbitals. The in-plane $\pi$ MOs are composed of $p$ atomic orbitals parallel to the ring $\left(p_{\text {in }}\right)$, while the out-of-plane $\pi$ MOs are composed of those perpendicular to the ring ( $p_{\text {out }}$ ). Fig. 4 illustrates how EEF polarizes the two kinds of $p$ orbitals on the carbon closest to the source of the EEF. Since the EEF is parallel to the axis of the $p_{\text {in }}$ atomic orbital, it can be expected that the polarization of the $p_{\text {in }}$ orbital should be easy to occur, because this does not require serious distortion of the orbital. We note that this kind of polarization is essentially caused by mixing of $2 s$ and $2 p_{\text {in }}$ atomic orbitals due to the EEF operator. ${ }^{[25]}$ In contrast, since the EEF is perpendicular to the $p_{\text {out }}$ atomic orbital, in order to polarize it the EEF must severely bend its shape, this is obviously more difficult to happen. So, from the orbital deformation point of view, the $\pi_{\text {out }}$ electrons are not as polarizable as the $\pi_{\text {in }}$ ones by the EEF parallel to the ring.
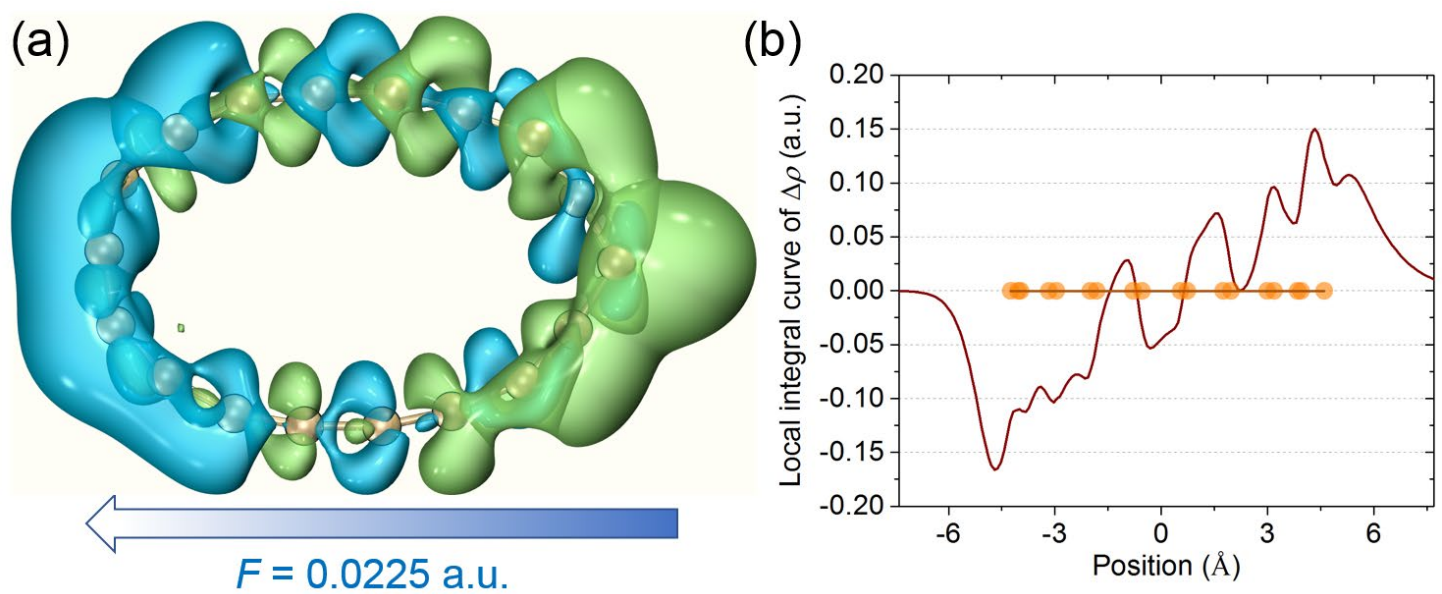

Fig. 5 Electron density polarization caused by EEF of 0.0225 a.u. (a) Isosurface map of electron density difference $(\Delta \rho)$ before and after applying EEF. Isovalue is set to be 0.001 a.u. Green and blue correspond to positive and negative parts, respectively (b) Local integral curve of the $\Delta \rho$, orange points highlight positions of atoms.

In order to intuitively and fully exhibit the charge polarization effect on the cyclo[18]carbon caused by the EEF, we calculated difference of electron density $(\Delta \rho)$ before and after applying EEF of 0.0225 a.u. based on the structure optimized under this field. Fig. 5(a) displays isosurface map of the $\Delta \rho$, while local integral curve of the $\Delta \rho$ is plotted as Fig. 5(b), which is defined as follows and able to quantify amount of $\Delta \rho$ in different slices

$$
I_{\text {local }}(x)=\int_{-\infty}^{+\infty} \int_{-\infty}^{+\infty} \Delta \rho(x, y, z) \mathrm{d} y \mathrm{~d} z
$$

From Fig. 5 one can clearly see that presence of EEF leads to significant charge polarization. More specifically, although the regions of increasing and decreasing electron density alternate with each other, in general the electrons greatly move towards the source of EEF. This phenomenon comes 
from the fact that the cyclo[18]carbon possesses rich $\pi$ electrons with global delocalization and highly polarizable characters. The overall reorganization of electron density also results in great change in the distribution of molecular electrostatic potential (ESP). The ESP colored van der Waals (vdW) surface map of the cyclo[18]carbon at EEF $=0.0225$ a.u. is drawn as Fig. S4, from which it can be seen that on the vdW surface of this molecule, the regions with positive and negative ESP are substantially separated with each other, and the most negative ESP is as low as $-88 \mathrm{kcal} / \mathrm{mol}$ while the most positive ESP is as high as $106 \mathrm{kcal} / \mathrm{mol}$. This observation is in great contrast to the situation without EEF. As we showed in Ref. [12], cyclo[18]carbon itself only has a low overall polarity, and the range of ESP over its vdW surface is quite narrow (about $-2 \sim 8 \mathrm{kcal} / \mathrm{mol}$ ).

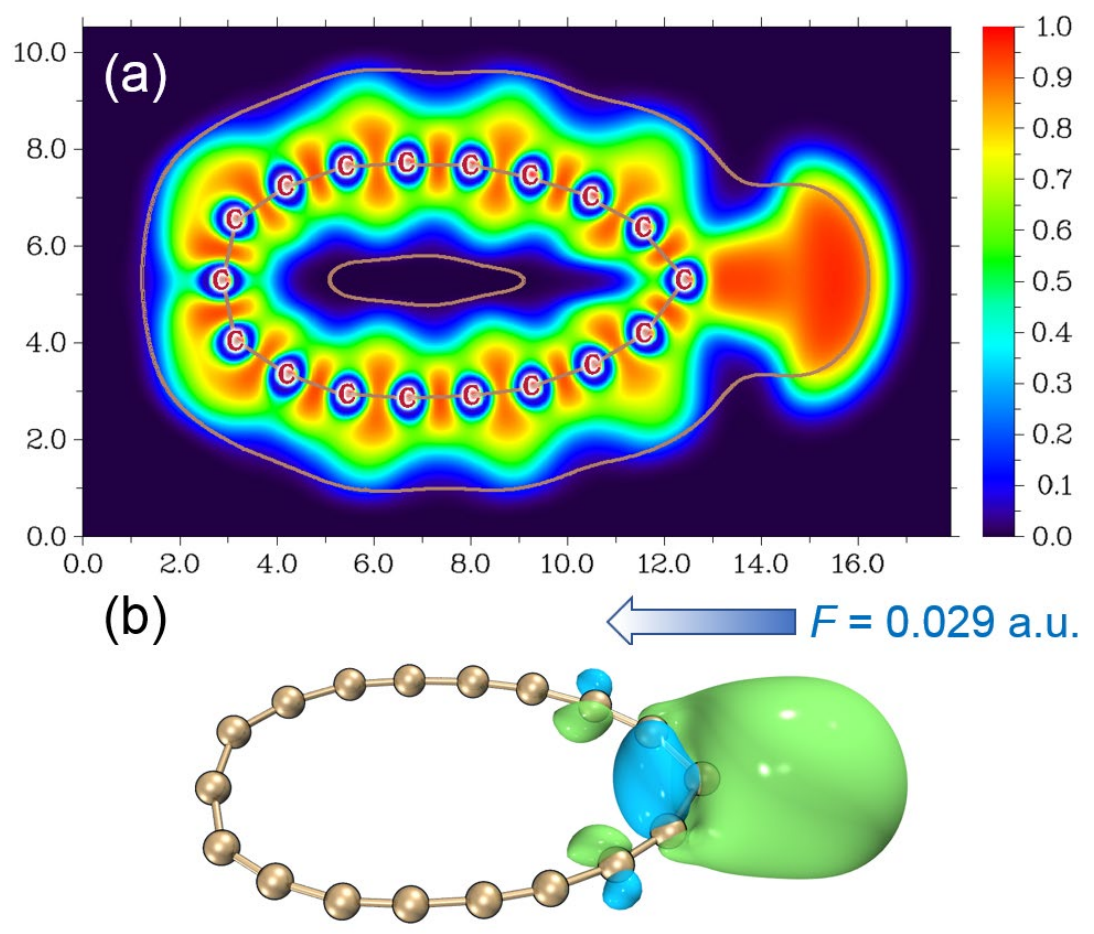

Fig. 6 Illustration of nearly detached electron of cyclo[18]carbon at EEF of 0.029 a.u. (a) Color-filled map of electron localization function in the ring plane. The brown contour line portrays electron density of 0.001 a.u. (b) Isosurface map of Foster-Boys localized orbital corresponding to the almost detached electron. Colors distinguish orbital phases and the isovalue is chosen to be 0.02 a.u.

As mentioned earlier, at limit EEF of 0.029 a.u., electron detachment from cyclo[18]carbon has almost occurred. Electron localization function (ELF) is a popular real space function that able to faithfully reveal localized electron, ${ }^{[41-44]}$ it was employed by us to graphically exhibit the occurrence region of the nearly detached electron. Fig. 6(a) displays ELF on the cyclo[18]carbon ring plane at $\mathrm{EEF}=0.029$ a.u., from which it can be seen that there is a large high electron localization region on the right side of the figure, its presence is a strong evidence of existence of very weakly bounded electron. It can be expected if the strength of the EEF is further increased, the highly polarized electron in this region should be fully detached from the system and move towards 
the source of the EEF. Orbital localization is another way of revealing the regions where localized electrons occur, ${ }^{[44,45]}$ it provides a complementary picture to ELF. We carried out orbital localization transformation via Foster-Boys method ${ }^{[46]}$ and indeed found an occupied localized molecular orbital that clearly represents the nearly unbounded electrons, see Fig. 6(b). This orbital has a nodal plane perpendicular to the ring plane, thus it essentially corresponds to the in-plane $\pi$ electrons that extremely polarized by EEF. This phenomenon is the direct consequence that the in-plane $\pi$ electrons in the cyclo[18]carbon can be remarkably polarized by the EEF applied parallelly to the ring plane, as we discussed above.

\section{Underlying causes of ring deformation under EEF: Energy variation and atomic forces}

In this section, we analyze the underlying sources making strong structural deformation of the cyclo[18]carbon under EEF in depth. In order to shed light on this point from an energy point of view, it is helpful to decompose the energy variation of the system during applying EEF as the following components

$$
E\left[\psi^{0}\left(\mathbf{R}^{0}\right), \widehat{H}^{0}\right] \stackrel{\Delta E_{\mathrm{def}}}{\longrightarrow} E\left[\psi^{0}\left(\mathbf{R}^{F}\right), \widehat{H}^{0}\right] \stackrel{\Delta E_{\text {int }}}{\longrightarrow} E\left[\psi^{0}\left(\mathbf{R}^{F}\right), \widehat{H}^{F}\right] \stackrel{\Delta E_{\text {relax }}}{\longrightarrow} E\left[\psi^{F}\left(\mathbf{R}^{F}\right), \widehat{H}^{F}\right]
$$

with $\psi, \mathbf{R}, \widehat{H}$ being electronic wavefunction, coordinate and Hamiltonian operator, respectively. Superscript of 0 and $F$ correspond to the situations without and with application of EEF, respectively. The $\Delta E_{\text {def }}$ reflects deformation energy of system structure due to EEF, the $\Delta E_{\text {int }}$ exhibits interaction energy between unrelaxed density and EEF at deformed structure, while $\Delta E_{\text {relax }}$ represents energy lowering due to relaxation of electron density under EEF. Obviously, the total energy variation can be expressed as

$$
\Delta E_{\text {total }}=E\left[\psi^{F}\left(\mathbf{R}^{F}\right), \widehat{H}^{F}\right]-E\left[\psi^{0}\left(\mathbf{R}^{0}\right), \widehat{H}^{0}\right]=\Delta E_{\text {def }}+\Delta E_{\text {int }}+\Delta E_{\text {relax }}
$$

Details of definition and evaluation of above terms are given in Section S2 in supplemental material. The results are presented in Fig. 7. 


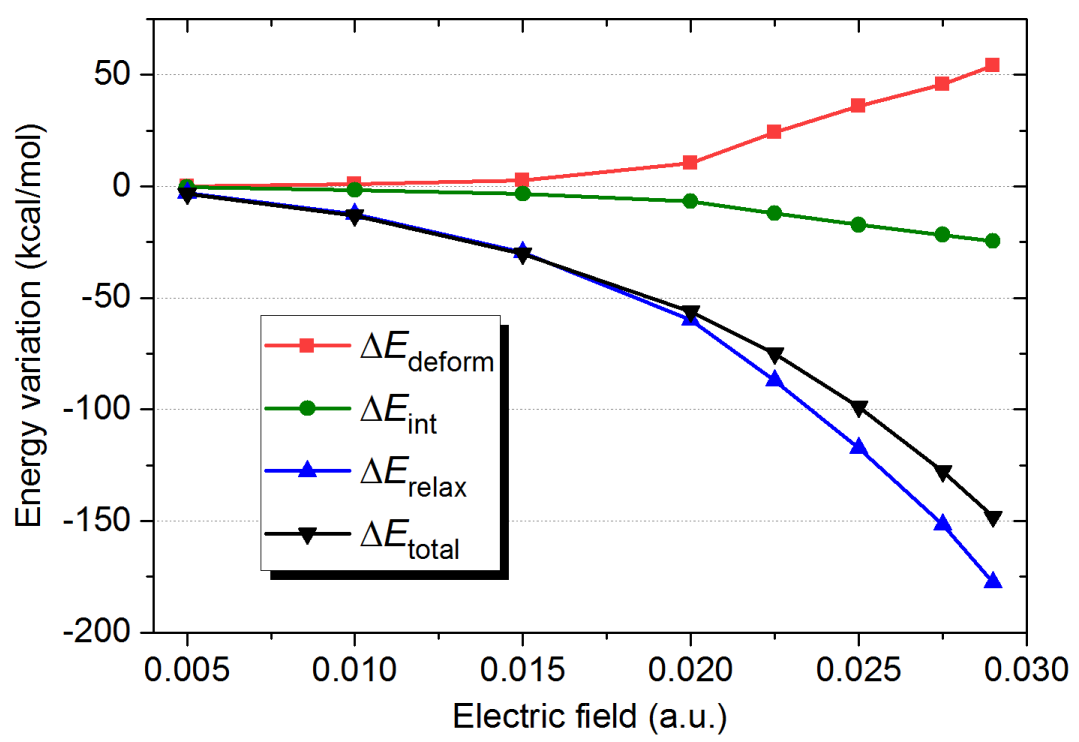

Fig. 7 Variation of total energy and its components of cyclo[18]carbon after applying EEFs of different magnitudes.

From Fig. 7 it can be seen that the energy consumed for deforming geometry of cyclo[18]carbon is modest when EEF $<0.02 \mathrm{a} . \mathrm{u}$, this is in line with the fact that the ring skeleton of this molecule has high flexibility. ${ }^{[12]}$ In the case of larger EEFs, although the $\Delta E_{\text {def }}$ reaches tens of $\mathrm{kcal} / \mathrm{mol}$ due to severe geometry deformation (see Fig. 1), its magnitude is still far smaller than

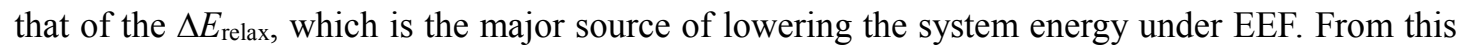
observation, the reason why EEF can markedly deform cyclo[18]carbon now become clearer, namely the highly polarizable $\pi$ electrons of this system can significantly relax under the EEF and thus greatly stablize the system, this effect is enough to overcome the energy penalty caused by the large structural deformation. We stress that this phenomenon is a very special character of the cyclo[18]carbon due to its special electronic and geometric structures. The $\Delta E_{\text {int }}$ shown in Fig. 7 plays a relatively unimportant role, it essentially equals to the stabilization interaction between the permanent dipole moment at deformed structure and EEF, and can be expressed as $\Delta E_{\text {int }}=$ $-\boldsymbol{\mu}\left[\psi^{0}\left(\mathbf{R}^{F}\right)\right] \cdot \mathbf{F}$, where $\mathbf{F}$ denotes EEF vector. It is worth to note that although permanent dipole moment of the cyclo[18]carbon itself is zero, it becomes absolutely non-negligible at the deformed structures induced by EEF. From the calculated data in Fig. S5 it can be see that the permanent dipole moment at the structure corresponding to $\mathrm{EEF}=0.025$ a.u. can even reach as high as 2.8 Debye (1.102 a.u.), which corresponds to the $\Delta E_{\text {int }}$ of $-1.102 \times 0.025=-0.0275$ Hartree $(-17.3$ $\mathrm{kcal} / \mathrm{mol})$.

The actual geometry relaxation process of the cyclo[18]carbon due to the presence of EEF is of theoretically interest. In order to explore this point, we generated a downhill trajectory under a large EEF (0.0275 a.u.) starting from the original geometry of cyclo[18]carbon, see Section S3 for the plot of energy and geometry variations in this process as well as relevant details. From which it can be seen that when EEF is just applied, the energy quickly decreases but the ring is not 
immediately elongated. In fact, at the initial stage, the geometry variation mainly corresponds to adjustment of some bond lengths and angles. After that, the length of the cyclo[18]carbon along the EEF direction rises steadily.

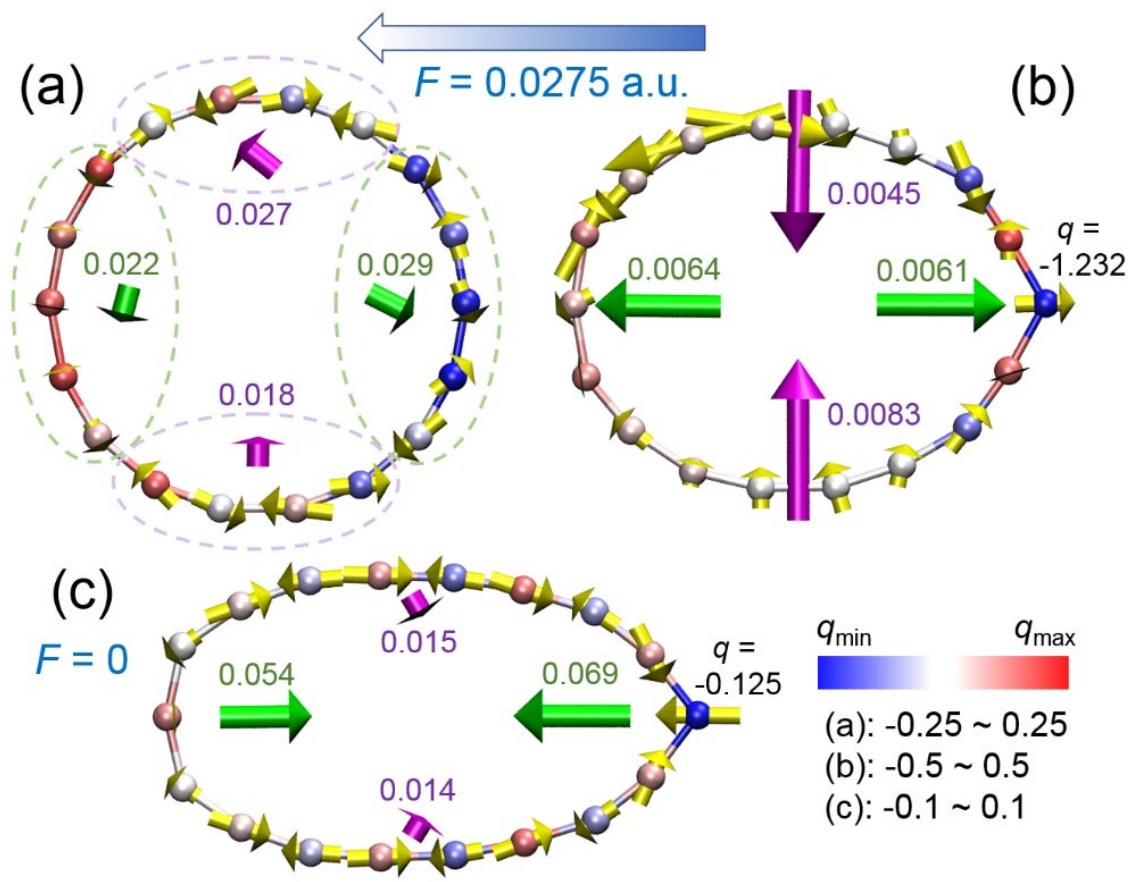

Fig. 8 Atomic forces and atomic charges of three representative status of cyclo[18]carbon. (a) Original structure with $\mathrm{EEF}=0.0275$ a.u. (b) Middle structure in downhill trajectory with $\mathrm{EEF}=0.0275$ a.u. (c) Structure optimized under EEF $=0.0275$ a.u. but with removal of EEF in the current case. Atomic forces are represented by yellow arrows, in each map the two green arrows respectively show collective forces of the atoms at the left and right sides of the system, while the two purple arrows respectively show that for the atoms at the top and bottom sides. Since different cases employ different scale factors of forces for clearly showing arrows, therefore values of collective forces are also labelled (in Hartree/Bohr). The lower and upper limits of color bar used for the three different cases are shown at lower right side.

It is interesting to look at the EEF induced ring deformation phenomenon from the perspective of forces, which is the most direct factor that drives structural deformation. The force on an atom due to EEF can be expressed as $\mathbf{f}=q \mathbf{F}$, where $q$ stands for atomic charge. It is easy to comprehend that application of EEF will break symmetric charge distribution of the cyclo[18]carbon and thus make its atomic charges no longer zero, which must in turn lead to atomic forces due to the interaction with EEF and the subsequent geometry distortion. To intuitively illustrate this point, for three representative status we drew forces as arrows and colored atoms according to their atomic charges, see Fig. 8. Atomic dipole moment corrected Hirshfeld (ADCH) method was employed for evaluating the atomic charges due to its good reliability and chemical meaning. ${ }^{[37,47]}$ From Fig. 8 we have the following noteworthy findings:

(1) In the presence of EEF, most atoms close to the EEF source have a negative charge, while 
most atoms far away from the source have a positive charge. This polarization effect is consistent with the phenomenon observed from the density difference map shown in Fig. 5. According to Fig. $8(c)$ and the corresponding range of color scale, it can be found that at the structure optimized under the EEF, charge unsymmetric distribution is still maintained even after removal of the EEF, though the extent has been significantly weakened. This point is in line with the discussion of permanent dipole moments above.

(2) At the original structure shown in Fig. 8(a), from the force it can be seen that application of EEF does not immediately cause the system to change from a circle to an ellipse, this observation agrees with the previous discussion on the downhill path.

(3) When the cyclo[18]carbon has undergone a certain degree of structural relaxation under EEF, as shown by the collective forces in Fig. 8(b), the system has a clear tendency to further elongate along the direction of the EEF, and meantime there is a clear tendency to squeeze the system in the direction perpendicular to the EEF. These forces make the deviation of the carbon ring from circular shape more and more prominent. In particular, the rightmost atom in the figure has a very obvious negative charge (-1.232), which certainly makes it to bear a huge force toward the source of the EEF, this should be the reason why its corresponding C-C-C bond angle $\left(121.2^{\circ}\right)$ is the smallest and most distorted one compared to the original structure $\left(160.0^{\circ}\right)$.

(4) It can be seen from Fig. 8(c) that after the structure of cyclo[18]carbon has been sufficiently polarized by a strong EEF, if the EEF is then removed, the atoms at both ends of longest axis of the system will receive a significant force toward the center of the system so that the system can quickly recover to a circle, suggesting that the cyclo[18]carbon has evident elastic characteristics. The elasticity of this system has also been extensively studied very recently in Ref. [11].

\section{Electric field regulation on HOMO-LUMO gap and optical absorption}

We next examine how EEF regulates energies of frontier molecular orbitals and optical absorption, both of them are quite important properties of chemical systems. Fig. 9 shows variation of HOMO and LUMO energies as well as their difference, namely HOMO-LUMO gap, which is closely related to molecular conductance. ${ }^{[48-50]}$ From the figure it can be seen that the EEF has a certain influence on the LUMO energy if the magnitude of EEF is small. When the EEF exceeds about 0.02 a.u., the energy of LUMO becomes quite sensitive to the field strength, and it decreases rapidly as the EEF strength increases. The reason of this observation can be understood from the orbital distribution. From the isosurface map of LUMO shown in Fig. S6, it can be seen that when the EEF strength is small, for example 0.015 a.u., the LUMO is an out-of-plane $\pi$ orbital and has not been notably polarized yet, just like the situation when there is no EEF. If the EEF increases to approximately 0.02 a.u. or higher, LUMO has changed to an in-plane $\pi$ orbital, and it expands conspicuously towards the source of the EEF. At a stronger EEF (0.029 a.u.), LUMO has almost completely separated from the molecule, implying that in this case system can no longer stably bind 
an additional electron. Since the energy of HOMO is relatively stable to the magnitude of the EEF, the variation character of the HOMO-LUMO gap with respect to field strength is similar to that of the LUMO energy, namely HOMO-LUMO gap narrows as EEF increases, and this effect is particularly prominent when EEF is large.

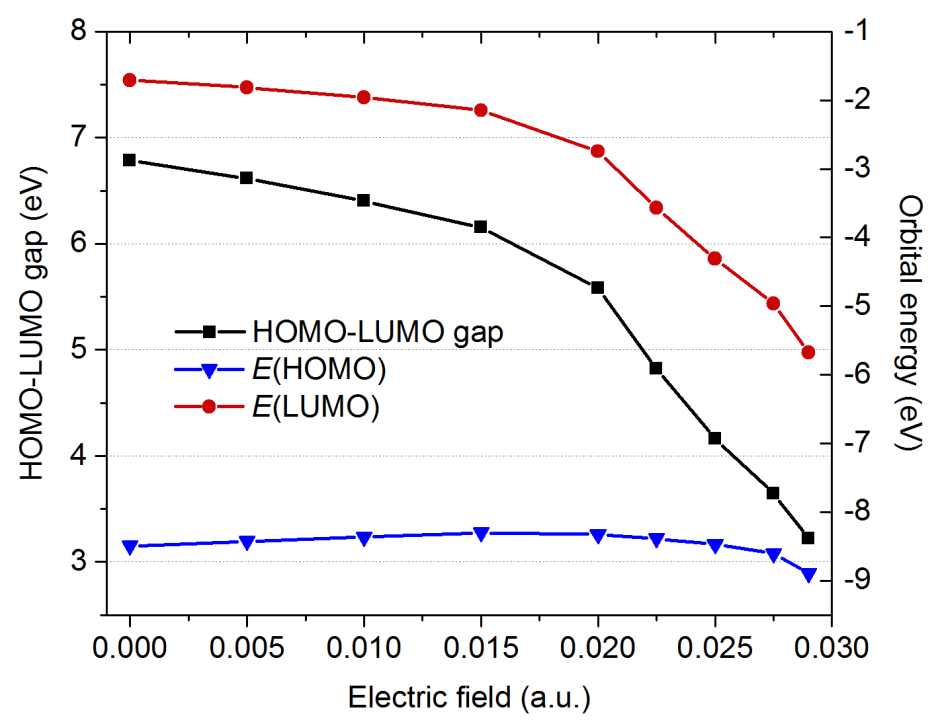

Fig. 9 Electric field regulation effect on HOMO energy, LUMO energy and HOMO-LUMO gap of cyclo[18]carbon.

In Ref. [13] we have showed that the cyclo[18]carbon does not have absorption in the visible light region but has an extremely strong absorption peak in the ultraviolet region, is this still true when EEF is present? To investigate this point, we calculated excitation energies and oscillator strengths for the excited states of the cyclo[18]carbon under different EEF strengths using timedependent density functional theory (TDDFT), and then simulated electronic absorption spectrum using Gaussian broadening function with full width at half maximum of $2 / 3 \mathrm{eV}$, the resulting spectra are collectively shown in Fig. 10. It can be seen that when EEF is small, the spectral characteristics do not change much compared to the situation without EEF, namely basically there is only a very strong absorption near $200 \mathrm{~nm}$. Starting from EEF of about 0.02 a.u., with the increase of the EEF, the very strong absorption gradually weakens, while an obvious new absorption band appears in the visible light region. The stronger the EEF, the higher the wavelength of this new band and the larger the absorption intensity. Therefore, it can be expected that the cyclo[18]carbon will show color when the applied EEF is large enough, and the color can be freely controlled by application of EEF with different strengths. 


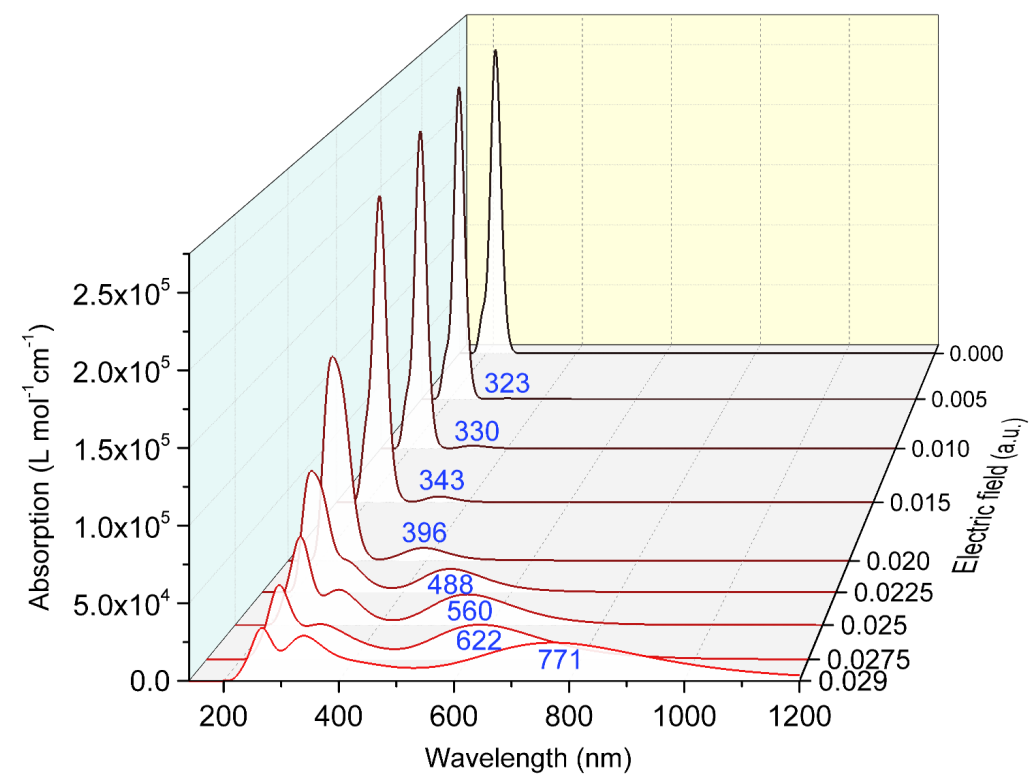

Fig. 10 Electric field regulation effect on electronic absorption spectrum of cyclo[18]carbon. The peak positions of the new absorption band induced by EEF are labelled as blue texts.

How to understand the evident EEF regulation effect on the electronic absorption spectrum of the cyclo[18]carbon? We first try to explore this problem from the perspective of orbital energy. The 10 highest occupied molecular orbitals (MOs) and 10 lowest unoccupied ones of the cyclo[18]carbon at different EEFs are given in Fig. S7, from which one can find that the influence of EEF on energies of occupied MOs is weak, while EEF noticeably decreases the energies of all low-lying unoccupied MOs. In particular, when EEF is large enough, then LUMO could be significantly lowered. Since under the TDDFT framework, electronic excitation can be formally represented as a batch of transitions from occupied MOs to unoccupied ones, the reason why EEF overall decreases wavelength of the strong absorption near $200 \mathrm{~nm}$ is somewhat explained.

It is worth to further unveil the nature of the obvious absorption band in the visible light region induced by a large EEF. Here we take the case of $\mathrm{EEF}=0.0275$ a.u. as a representative example for analysis. We first decomposed the total absorption spectrum curve in this case, the respective contribution curves of the electronic excitations with oscillator strength greater than 0.1 are plotted in Fig. S8. It can be seen that the absorption band with maximum absorption at $621.8 \mathrm{~nm}$ induced by the EEF is mainly caused by $\mathrm{S} 0 \rightarrow \mathrm{S} 6$ excitation, which was found to be primarily (91\%) contributed by the transition from HOMO-3 to LUMO. Therefore, we further plotted the isosurface maps of the two orbitals to examine their characteristics, see Fig. 11. The HOMO-3 is a marginally polarized in-plane $\pi$ orbital, while LUMO is an orbital almost separated from the molecule due to remarkable induction of EEF. Because the LUMO in this form only appears under a strong EEF, and there is an evident spatial overlap between the LUMO and HOMO-3, and moreover the transition between these two orbitals is not spatially forbidden, the reason why EEF can induce such an obvious absorption band is clearly understandable. Moreover, since the energy of LUMO in the 
current status is very low, it is reasonable to expect that the excitation energy should be small and thus the corresponding absorption fall in the visible light region.

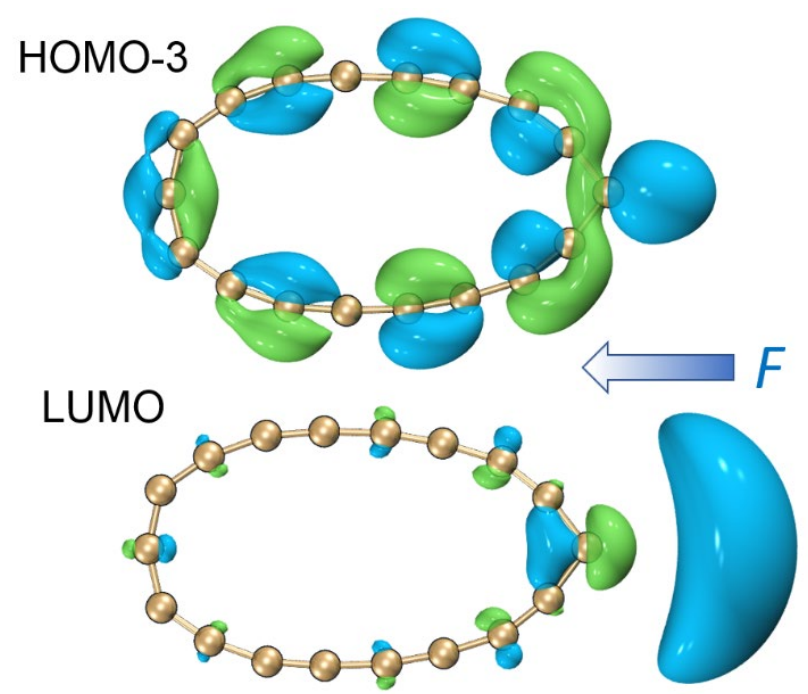

Fig. 11 Isosurface map of HOMO-3 and LUMO of cyclo[18]carbon corresponding to the case of EEF = 0.0275 a.u. Isovalue is chosen to be 0.03 . The positive and negative orbital phases are distinguished by the two colors.

\section{Realizing electric field regulation effect in a chemical way}

As discussed above, although EEF has a great influence on various aspects of the cyclo[18]carbon, only the strong EEF (>0.02 a.u.) has a particularly significant effect on its geometric structure and UV-Vis spectrum. Unfortunately, high intensity EEF is not easy to achieve via current physical technology. So, is there any easier way to realize the effect similar to strong EEF? We noticed that alkali metal and alkaline earth metal cations can generate a strong EEF on the surroundings, and they can only combine with the cyclo[18]carbon through non-covalent interaction without evidently destroying its original electronic structure. Therefore, it is expected that introduction of these cations to the cyclo[18]carbon can cause a similar influence as directly applying a strong EEF. Obviously, achieving EEF regulation effect on the cyclo[18]carbon in this chemical way is more likely to be widely used in the future.

To verify this idea, we respectively optimized the complexes composed of a cyclo[18]carbon molecule and various cations. $\mathrm{Li}^{+}, \mathrm{Na}^{+}, \mathrm{K}^{+}, \mathrm{Be}^{2+}, \mathrm{Mg}^{2+}$ and $\mathrm{Ca}^{2+}$ were taken into account. The geometries of the two representative complexes $\mathrm{C}_{18} @ \mathrm{Na}^{+}$and $\mathrm{C}_{18} @ \mathrm{Mg}^{2+}$ are shown in Fig. 12, while others are given in Fig. S9. From the figures it can be clearly seen that the monovalent cations are unable to effectively polarize structure of the cyclo[18]carbon, the variations of the longest axis of the carbon ring are all lower than $0.1 \AA$. In contrast, the divalent cations deform the carbon ring remarkably, and we note the smaller the cation radius, that is the higher the density of the positive charge, the stronger the polarization effect. The order of increase of the longest axis of the 
cyclo[18] carbon owing to the cations is $\mathrm{Be}^{2+}(0.92 \AA)>\mathrm{Mg}^{2+}(0.85 \AA)>\mathrm{Ca}^{2+}(0.41 \AA)$. By comparing the geometric variation of the ring under different EEFs (Fig. S1), it is anticipated that $\mathrm{Be}^{2+}$ and $\mathrm{Mg}^{2+}$ can approximately equivalently apply about 0.02 a.u. of EEF to the cyclo[18]carbon, and thus it is expected they could largely influence optical absorption of the cyclo[18]carbon. It is worth to note that there are partial electron transfers from the carbon ring to the cations. As indicated by the atomic charge labelled on Figs. 12 and S9, the extent of electron transfer is $\mathrm{Be}^{2+}>\mathrm{Mg}^{2+}>$ $\mathrm{Ca}^{2+}>\mathrm{Li}^{+}>\mathrm{Na}^{+}>\mathrm{K}^{+}$, this order is in line with their abilities of polarizing surroundings.
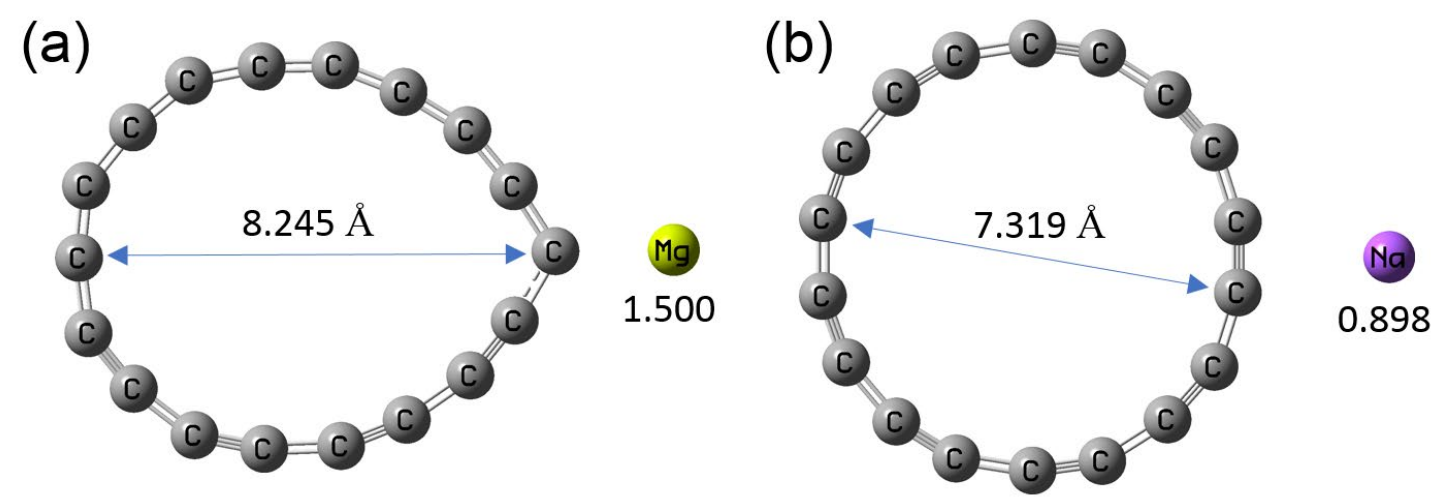

Fig. 12 Optimized geometry of (a) $\mathrm{C}_{18} @ \mathrm{Mg}^{2+}$ and (b) $\mathrm{C}_{18} @ \mathrm{Na}^{+}$complexes. The lengths of the longest axis of the cyclo[18]carbon moieties are labelled. ADCH atomic charges of the cations are indicated.

Electronic absorption spectra of the cyclo[18]carbon combined with various divalent cations are shown in Fig. 13. We can see that all cations reduce the intensity of the absorption band in the ultraviolet region, while new absorption bands appear in the visible light region, indicating that these ions have approximately shown a strong EEF effect on the cyclo[18]carbon. It is especially worth mentioning that the absorption spectrum of the $\mathrm{C}_{18} @ \mathrm{Mg}^{2+}$ is very similar to that of the cyclo[18]carbon under EEF of 0.02 a.u. Specifically, the EEF of 0.02 a.u. causes an additional absorption peak at $396 \mathrm{~nm}$ (Fig. 10), while $\mathrm{Mg}^{2+}$ induces a new absorption peak at $378 \mathrm{~nm}$ with comparable absorption strength. It can also be seen from Fig. 13 that $\mathrm{C}_{18} @ \mathrm{Be}^{2+}$ also leads to an absorption band with peak at $575 \mathrm{~nm}$, so its displayed color must be significantly different from that of $\mathrm{C}_{18} @ \mathrm{Mg}^{2+}$ and $\mathrm{C}_{18} @ \mathrm{Ca}^{2+}$. The absorption spectra of $\mathrm{C}_{18} @ \mathrm{Li}^{+}, \mathrm{C}_{18} @ \mathrm{Na}^{+}$and $\mathrm{C}_{18} @ \mathrm{~K}^{+}$are shown in Fig. S10, which clearly shows that the monovalent cations are not able to sufficiently polarize the cyclo[18]carbon to result in detectable absorption band in the visible light region, most because their electric fields are not strong enough. 


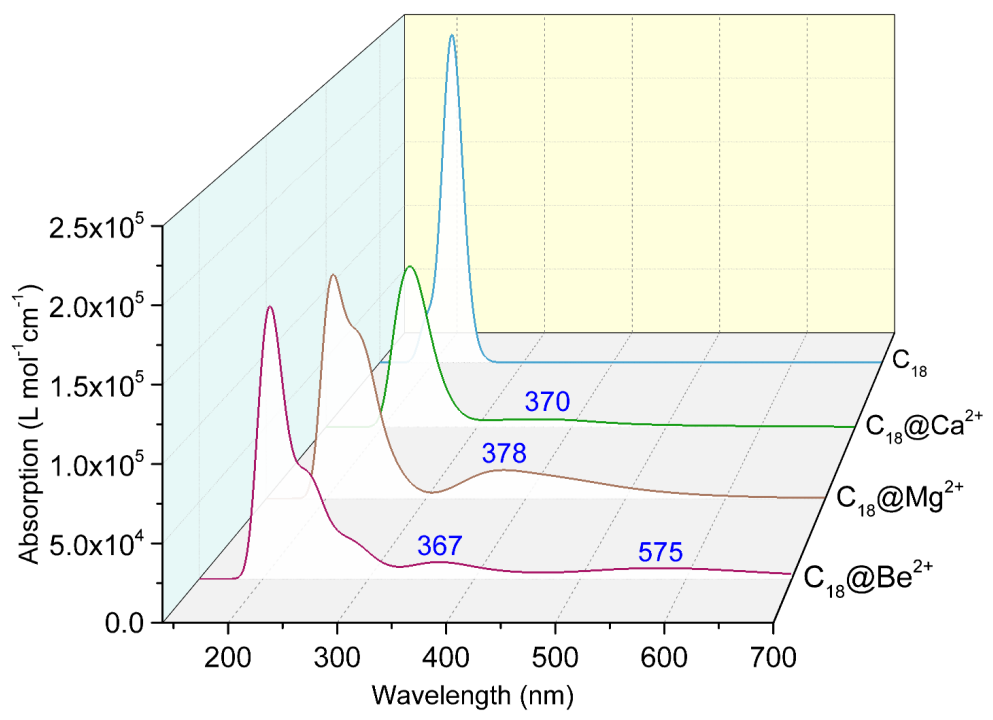

Fig. 13 Electronic absorption spectrum of cyclo[18]carbon and its complexes with various divalent cations. Peak positions of the new absorption band induced by the cations are labelled as blue texts.

It is interesting to explore the nature of the absorption peak at $378 \mathrm{~nm}$ of the $\mathrm{C}_{18} @ \mathrm{Mg}^{2+}$. This peak was found to be mostly contributed by $\mathrm{S} 0 \rightarrow \mathrm{S} 15$ excitation, which is strongly mixed by many MO transitions. In order to vividly exhibit the character of this excitation, we employed the holeelectron analysis proposed by us, ${ }^{[13,51]}$ which is able to rigorously represent an electron excitation as a "hole" to "electron" transition. Distributions of hole and electron corresponding to the $\mathrm{S} 0 \rightarrow \mathrm{S} 15$ excitation are plotted in Fig. 14 as blue and green isosurfaces, respectively. It can be seen that this excitation shows conspicuous charge transfer character, that is during the excitation an electron is almost completely transfered from the cyclo[18]carbon to the $\mathrm{Mg}^{2+}$ cation. This excitation character is akin to that shown in Fig. 11, further demonstrating that addition of an alkaline earth metal ion can have a similar effect to the application of a strong EEF. Now we can conclude that it is possible to approximately achieve the regulation effect of EEF on the cyclo[18]carbon in terms of introducing a proper cation.

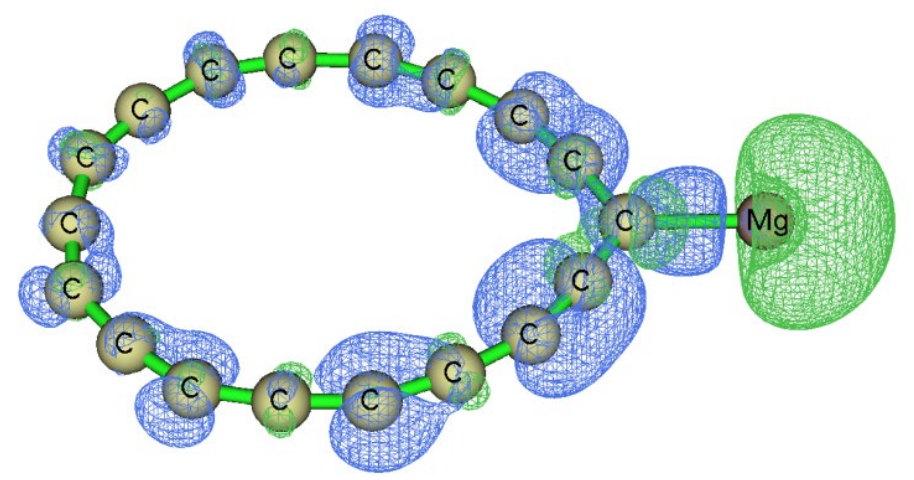

Fig. 14 Hole (blue) and electron (green) isosurfaces of $\mathrm{S} 0 \rightarrow \mathrm{S} 15$ excitation of cyclo[18]carbon combined with $\mathrm{Mg}^{2+}$ cation. Isovalue is set to 0.002 . 


\section{Summary}

In this article, through theoretical calculations, we comprehensively studied how EEF affects various aspects of the novel cyclo[18]carbon system and gave detailed explanations on the observed phenomena. This work mainly has the following findings

(1) EEF is able to cause evident structure deformation of the cyclo[18]carbon. Especially, when the EEF is large enough ( $c a .0 .02$ a.u. or $1 \mathrm{~V} / \AA$ ), the ring will be significantly elongated in the direction of the applied field and thus change from a circular shape to an obvious ellipse.

(2) EEF significantly polarizes charge distribution of the cyclo[18]carbon. As the strength of the EEF increases, the dipole moment of the system increases sharply. The main contributor to the induced dipole moment is the in-plane $\pi$ electrons, which delocalize parallelly to the ring plane and can be strongly polarized by EEF. In contrast, the degree of polarization of out-of-plane $\pi$ electrons by the EEF is much lower.

(3) When the magnitude of applied EEF is increased to about 0.029 a.u., a portion of in-plane $\pi$ electrons will nearly detach from the cyclo[18]carbon. If the EEF is further enhanced, the molecule is ionized.

(4) The larger the EEF, the smaller the HOMO-LUMO gap. This is mainly because the presence of the EEF greatly reduces energy of LUMO, while energies of occupied orbitals are relatively less affected.

(5) EEF notably affects the optical absorption characteristics of the cyclo[18]carbon. When the EEF exceeds about 0.02 a.u., as the field strength increases, the intensity of the very strong absorption peak in the ultraviolet region weakens and the wavelength decreases. In addition, the EEF also induces the system to appear an obvious absorption in the visible light region, which is found to be mainly caused by the transition from an in-plane $\pi$ orbital to the extremely polarized LUMO.

In addition, we discussed the underlying energy variation of the cyclo[18]carbon under the EEF through an energy decomposition analysis, and we also investigated the ring deformation and ring recovery from the perspective of atomic force. These analyses help to deeply understand the driven force of ring deformation induced by EEF.

Considering that it is not quite easy to achieve a large EEF in current experimental technology, we also explored the possibility of equivalently applying strong EEF on the cyclo[18]carbon in a more feasible way. We demonstrated that addition of divalent alkaline earth metal cation can play a similar role as strong EEF, namely notably distorting structure of the cyclo[18]carbon and making it have optical absorption in visible light region.

Our findings in this work should not only help researchers to better understand response of this unusual system to EEF, but also provide a new path for the design of novel materials based on the cyclo[18]carbon and its derivatives; for example, it is seemingly promising to design deformable 
materials driven by EEF, and to design dyes whose color are tunable by strength of applied EEF or added cations.

\section{Supporting Information}

Effect of applying EEF in different directions; Details of evaluating components of energy variation under EEF; Downhill trajectory; Variation of length of the longest axis, bond angles and permanent dipole moment of cyclo[18]carbon with respect to EEF; Laplacian bond order; ESP mapped molecular vdW surface; Isosurface map of LUMO; Energies of frontier MOs; Electronic absorption spectra; Optimized geometries (PDF)

\section{References}

1. K. Kaiser; L. M. Scriven; F. Schulz; P. Gawel; L. Gross; H. L. Anderson. An sp-hybridized molecular carbon allotrope, cyclo[18]carbon. Science, 365, 1299 (2019).

2. M. M. Haley. Cyclo[18]carbon, the Newest Member of the Family of Carbon Allotropes. Chem, 5, 2517 (2019). DOI: 10.1016/j.chempr.2019.09.011.

3. M. Z. Rahman; T. Edvinsson. Rational design and resolution of the mystery of the structure of Cyclo[18]carbon. J. Mater. Chem. A, 8, 8234 (2020). DOI: 10.1039/C9TA13193K.

4. N. Fedik; M. Kulichenko; D. Steglenko; A. I. Boldyrev. Can aromaticity be a kinetic trap? Example of mechanically interlocked aromatic [2-5]catenanes built from cyclo[18]carbon. Chem. Commun., 56, 2711 (2020). DOI: 10.1039/C9CC09483K.

5. S. Hussain; H. Chen; Z. Zhang; H. Zheng. Vibrational spectra and chemical imaging of cyclo[18]carbon by tip enhanced Raman spectroscopy. Chem. Commun., 56, 2336 (2020). DOI: 10.1039/C9CC09130K.

6. A. J. Stasyuk; O. A. Stasyuk; M. Solà; A. A. Voityuk. Cyclo[18]carbon: the smallest all-carbon electron acceptor. Chem. Commun., 56, 352 (2020). DOI: 10.1039/C9CC08399E.

7. L. Zhang; H. Li; Y. P. Feng; L. Shen. Diverse Transport Behaviors in Cyclo[18]carbon-Based Molecular Devices. J. Phys. Chem. Lett., 11, 2611 (2020). DOI: 10.1021/acs.jpclett.0c00357.

8. Z. S. Pereira; E. Z. da Silva. Spontaneous Symmetry Breaking in Cyclo[18]Carbon. J. Phys. Chem. $A, \mathbf{1 2 4}, 1152$ (2020). DOI: 10.1021/acs.jpca.9b11822.

9. A. Nandi; E. Solel; S. Kozuch. Carbon Tunneling in the Automerization of Cyclo[18]carbon. Chem.-Eur. J., 26, 625 (2020). DOI: 10.1002/chem.201904929.

10. G. V. Baryshnikov; R. R. Valiev; A. V. Kuklin; D. Sundholm; H. Ågren. Cyclo[18]carbon: Insight into Electronic Structure, Aromaticity, and Surface Coupling. J. Phys. Chem. Lett., 10, 6701 (2019). DOI: 10.1021/acs.jpclett.9b02815.

11. S. Fang; Y. H. Hu. Cyclo[18]carbon as an Ultra-Elastic Molecular O-Ring with Unique Mechanical Properties. Carbon (2020). DOI: 10.1016/j.carbon.2020.08.082.

12. T. Lu; Q. Chen; Z. Liu. A thorough theoretical exploration of intriguing characteristics of cyclo[18]carbon: Geometry, bonding nature, aromaticity, weak interaction, reactivity, excited states, vibrations, molecular dynamics and various molecular properties. ChemRxiv (2019). DOI: 10.26434/chemrxiv.11320130. 
13. Z. Liu; T. Lu; Q. Chen. An sp-hybridized all-carboatomic ring, cyclo[18]carbon: Electronic structure, electronic spectrum, and optical nonlinearity. Carbon, 165, 461 (2020). DOI: 10.1016/j.carbon.2020.05.023.

14. Z. Liu; T. Lu; Q. Chen. An sp-hybridized all-carboatomic ring, cyclo[18]carbon: Bonding character, electron delocalization, and aromaticity. Carbon, 165, 468 (2020). DOI: 10.1016/j.carbon.2020.04.099. 15. T. Lu; Q. Chen. van der Waals potential: an important complement to molecular electrostatic potential in studying intermolecular interactions. ChemRxiv (2020). DOI: 10.26434/chemrxiv.12148572. 16. Z. Liu; T. Lu; Q. Chen. Intermolecular interaction characteristics of the all-carboatomic ring, cyclo[18]carbon: Focusing on molecular adsorption and stacking. Carbon (2020). DOI: 10.1016/j.carbon.2020.09.048.

17. T. Stuyver; D. Danovich; J. Joy; S. Shaik. External electric field effects on chemical structure and reactivity. WIREs Comput. Mol. Sci., 10, e1438 (2020). DOI: 10.1002/wcms.1438.

18. J. Chen; S. Liu; M. Li; C. Rong; S. Liu. A density functional theory and information-theoretic approach study of chiral molecules in external electric fields. Chem. Phys. Lett., 757, 137858 (2020). DOI: $10.1016 /$ j.cplett.2020.137858.

19. Z.-J. Zhou; X.-P. Li; Z.-B. Liu; Z.-R. Li; X.-R. Huang; C.-C. Sun. Electric Field-Driven Acid-Base Chemistry: Proton Transfer from Acid $(\mathrm{HCl})$ to Base (NH3/H2O). J. Phys. Chem. A, 115, 1418 (2011). DOI: $10.1021 /$ jp110408y.

20. T. James; D. J. Wales; J. Hernández Rojas. Energy landscapes for water clusters in a uniform electric field. J. Chem. Phys., 126, 054506 (2007). DOI: 10.1063/1.2429659.

21. Y.-H. Yin; Q. Wang. The electric fields enhance the non-covalent intermolecular interaction between H2 and (MgO)3. Chem. Phys., 539, 110925 (2020). DOI: 10.1016/j.chemphys.2020.110925.

22. X. Zhang; Y. Liu; X. Ma; F. Jin; B. Abulimiti; M. Xiang. Tuning the optical properties of (TiO2)2 via the electric field. Optik, 221, 165395 (2020). DOI: 10.1016/j.ijleo.2020.165395.

23. L. Hao; J. Wang; D. Zhai; P. Ma; C. Ma; Y. Pan; J. Jiang. Theoretical Study on CL-20-Based Cocrystal Energetic Compounds in an External Electric Field. ACS Omega, 5, 14767 (2020). DOI: 10.1021/acsomega.0c01643.

24. Y. Tao; Q. Wang; K. Sun; Q. Zhang; W. Liu; J. Du; Z. Liu. The molecular structure, spectroscopic features and electronic properties of tioxolone under the external electric field. Spectrochim. Acta, Part A, 231, 118108 (2020). DOI: 10.1016/j.saa.2020.118108.

25. S. Shaik; R. Ramanan; D. Danovich; D. Mandal. Structure and reactivity/selectivity control by oriented-external electric fields. Chem. Soc. Rev., 47, 5125 (2018). DOI: 10.1039/C8CS00354H.

26. S. Shaik; D. Danovich; J. Joy; Z. Wang; T. Stuyver. Electric-Field Mediated Chemistry: Uncovering and Exploiting the Potential of (Oriented) Electric Fields to Exert Chemical Catalysis and Reaction Control. J. Am. Chem. Soc., 142, 12551 (2020). DOI: 10.1021/jacs.0c05128.

27. M. Wahadoszamen; T. Nakabayashi; S. Kang; H. Imahori; N. Ohta. External Electric Field Effects on Absorption and Fluorescence Spectra of a Fullerene Derivative and Its Mixture with ZincTetraphenylporphyrin Doped in a PMMA Film. J. Phys. Chem. B, 110, 20354 (2006). DOI: 10.1021/jp0635967.

28. Y.-W. Son; M. L. Cohen; S. G. Louie. Half-metallic graphene nanoribbons. Nature, 444, 347 (2006). DOI: $10.1038 /$ nature 05180 .

29. O. Hod; V. Barone; G. E. Scuseria. Half-metallic graphene nanodots: A comprehensive firstprinciples theoretical study. Phys. Rev. B, 77, 035411 (2008). DOI: 10.1103/PhysRevB.77.035411.

30. X.-Q. Tian; X.-R. Wang; Y.-D. Wei; L. Liu; Z.-R. Gong; J. Gu; Y. Du; B. I. Yakobson. Highly 
Tunable Electronic Structures of Phosphorene/Carbon Nanotube Heterostructures through External Electric Field and Atomic Intercalation. Nano Lett., 17, 7995 (2017). DOI: 10.1021/acs.nanolett.7b04562. 31. J. Klinovaja; M. J. Schmidt; B. Braunecker; D. Loss. Carbon nanotubes in electric and magnetic fields. Phys. Rev. B, 84, 085452 (2011). DOI: 10.1103/PhysRevB.84.085452.

32. M. Khazaei; A. A. Farajian; S. U. Lee; R. V. Belosludov; Y. Kawazoe. Carbon Nanotubes Oscillation under Electric Field. Jpn. J. Appl. Phys., 49, 115103 (2010). DOI: 10.1143/jjap.49.115103.

33. M. J. Frisch; G. W. Trucks; H. B. Schlegel; G. E. Scuseria; M. A. Robb; J. R. Cheeseman; G. Scalmani; V. Barone; G. A. Petersson; H. Nakatsuji; X. Li; M. Caricato; A. V. Marenich; J. Bloino; B. G. Janesko; R. Gomperts; B. Mennucci; H. P. Hratchian; J. V. Ortiz; A. F. Izmaylov; J. L. Sonnenberg; Williams; F. Ding; F. Lipparini; F. Egidi; J. Goings; B. Peng; A. Petrone; T. Henderson; D. Ranasinghe; V. G. Zakrzewski; J. Gao; N. Rega; G. Zheng; W. Liang; M. Hada; M. Ehara; K. Toyota; R. Fukuda; J. Hasegawa; M. Ishida; T. Nakajima; Y. Honda; O. Kitao; H. Nakai; T. Vreven; K. Throssell; J. A. Montgomery Jr.; J. E. Peralta; F. Ogliaro; M. J. Bearpark; J. J. Heyd; E. N. Brothers; K. N. Kudin; V. N. Staroverov; T. A. Keith; R. Kobayashi; J. Normand; K. Raghavachari; A. P. Rendell; J. C. Burant; S. S. Iyengar; J. Tomasi; M. Cossi; J. M. Millam; M. Klene; C. Adamo; R. Cammi; J. W. Ochterski; R. L. Martin; K. Morokuma; O. Farkas; J. B. Foresman; D. J. Fox. Gaussian 16 A.03, Wallingford, CT, 2016. 34. J.-D. Chai; M. Head-Gordon. Long-range corrected hybrid density functionals with damped atomatom dispersion corrections. Phys. Chem. Chem. Phys., 10, 6615 (2008). DOI: 10.1039/b810189b.

35. M. J. Frisch; J. A. Pople; J. S. Binkley. Self-consistent molecular orbital methods 25. Supplementary functions for Gaussian basis sets. J. Chem. Phys., 80, 3265 (1984).

36. R. Krishnan; J. S. Binkley; R. Seeger; J. A. Pople. Self-consistent molecular orbital methods. XX. A basis set for correlated wave functions. J. Chem. Phys., 72, 650 (1980).

37. T. Lu; F. Chen. Multiwfn: A Multifunctional Wavefunction Analyzer. J. Comput. Chem., 33, 580 (2012). DOI: $10.1002 / j c c .22885$.

38. Semichem, Inc., GaussView 6.0.16 program: http://gaussian.com/gaussview6/ (accessed on Aug 29, 2020).

39. W. Humphrey; A. Dalke; K. Schulten. VMD: Visual molecular dynamics. J. Mol. Graph., 14, 33 (1996). DOI: 10.1016/0263-7855(96)00018-5.

40. T. Lu; F. Chen. Bond Order Analysis Based on the Laplacian of Electron Density in Fuzzy Overlap Space. J. Phys. Chem. A, 117, 3100 (2013). DOI: 10.1021/jp4010345.

41. A. D. Becke; K. E. Edgecombe. A Simple Measure of Electron Localization in Atomic and Molecular Systems. J. Chem. Phys., 92, 5397 (1990). DOI: 10.1063/1.458517.

42. T. Lu; F. Chen. Meaning and Functional Form of the Electron Localization Function. Acta Phys. Chim. Sin., 27, 2786 (2011).

43. T. Lu; Q. Chen. Revealing Molecular Electronic Structure via Analysis of Valence Electron Density. Acta Phys. -Chim. Sin., 34, 503 (2018). DOI: 10.3866/pku.Whxb201709252.

44. T. Lu; Q. Chen. A simple method of identifying $\pi$ orbitals for non-planar systems and a protocol of studying $\pi$ electronic structure. Theor. Chem. Acc., 139, 25 (2020). DOI: 10.1007/s00214-019-2541-z.

45. J. Pipek; P. G. Mezey. A fast intrinsic localization procedure applicable for ab initio and semiempirical linear combination of atomic orbital wave functions. J. Chem. Phys., 90, 4916 (1989). DOI: $10.1063 / 1.456588$.

46. J. M. Foster; S. F. Boys. Canonical Configurational Interaction Procedure. Reviews of Modern Physics, 32, 300 (1960). DOI: 10.1103/RevModPhys.32.300.

47. T. Lu; F. Chen. Atomic Dipole Moment Corrected Hirshfeld Population Method. J. Theor. Comp. 
Chem., 11, 163 (2012). DOI: doi:10.1142/S0219633612500113.

48. J.-L. Bredas. Mind the gap! Mater. Horiz., 1, 17 (2014). DOI: 10.1039/C3MH00098B.

49. S. Manzetti; T. Lu; H. Behzadi; M. D. Estrafili; H.-L. Thi Le; H. Vach. Intriguing properties of unusual silicon nanocrystals. RSC Adv., 5, 78192 (2015). DOI: 10.1039/C5RA17148B.

50. S. Manzetti; T. Lu. Alternant conjugated oligomers with tunable and narrow HOMO-LUMO gaps as sustainable nanowires. $R S C A d v$, 3, 25881 (2013). DOI: 10.1039/c3ra41572d.

51. T. Lu. Multiwfn Manual, version 3.7, Section 3.21.1, available at http://sobereva.com/multiwfn (accessed on Sep 2, 2020). 
Table of Contents

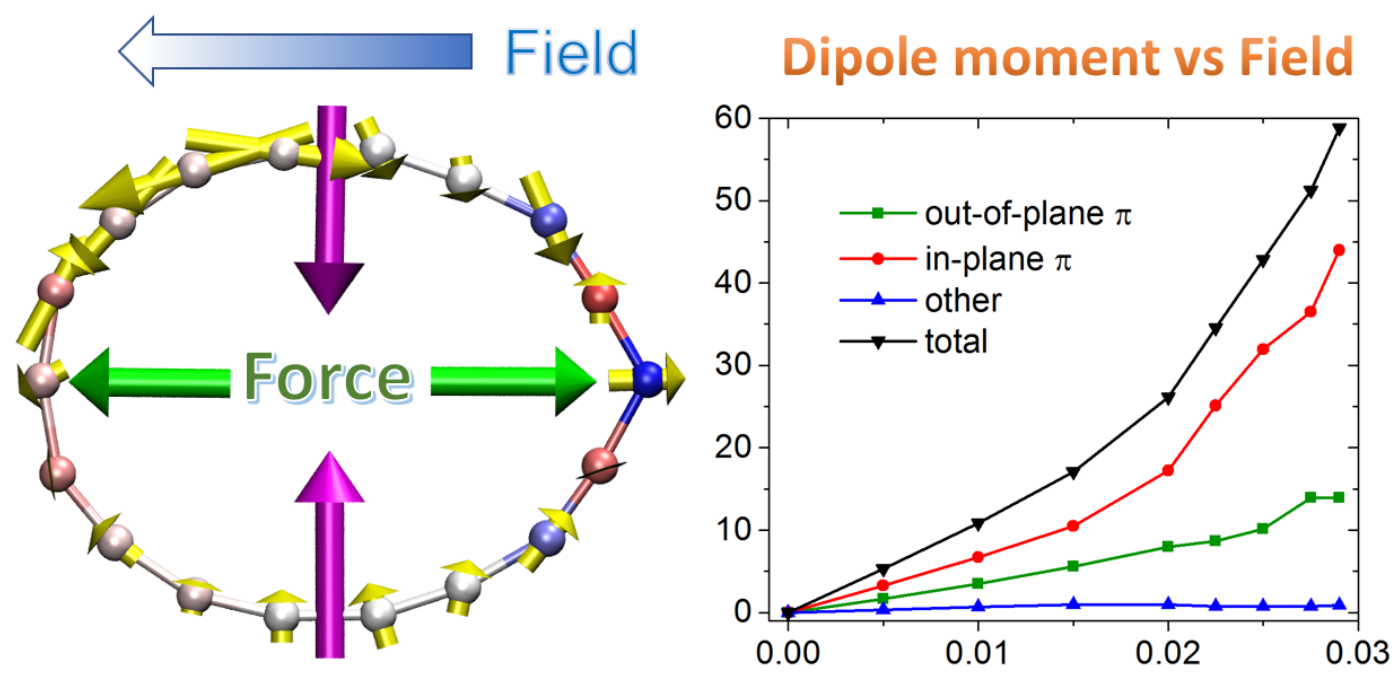

